(Aus dem physiologischen Institute der Universität Wien.)

\title{
Die motorischen Wurzelfasern für die Kardia und den Magen.
}

Von

Dr. Walter Zweig.

(Mit 1 Textfigur und Tafel XIV.)

Durch die Arbeiten von Grossmann ${ }^{1}$ ), Grabower ${ }^{2}$ ), Réthis ${ }^{3}$ ) und $\mathrm{Kreidl^{4 }}$ ) wurde mit verfeinerten Untersuchungsmethoden die Frage in Angriff genommen, welche Funktion die einzelnen Wurzelfasern haben, die, aus der Medulla oblongata austretend, den Glossopharyngeus, Vagus und Accessorius zusammensetzen. Nach dem Vorschlag von Grossmann empfiehlt es sich, die Wurzelfasern in ein oberes, mittleres und unteres Bündel zu trennen, wobei das obere wesentlich dem Glossopharyngeus, das mittlere dem Vagus und das untere dem Medullarteil des Accessorius der Anatomen entspricht.

Aus dem oberen Bündel stammen nach Grossmann die zum M. cricothyrenideus und die im Vagusstamm zu den Lungen ziehenden Hering-Breuer'schen Fasern. Grabower und Grossmann fanden, dass die übrigen zum Kehlkopf fübrenden Nerven aus dem mittleren Bündel hervorgehen.

1) Grossmann, Über die A tembewegungen des Kehlkopfes. II. Teil. Die Wurzelfasern der Kehlkopfnerven. Sitzungsber. d. k. Akademie der Wissenschaft Bd. 98 Abt. III. 1889.

2) Grabower, Das Wurzelgebiet der motorischen Kehlkopfnerven. Zentralblatt f. Physiol. Bd. 3. 1890.

3) L. Réthi, Der periphere Verlauf der motorischen Rachen- und Gaumennerven. Sitzungsber. d. k. Akademie der Wissensch. Bd. 102 S. 10 Abt. 3. 1893.

4) Kreidl, Die Wurzelfasern der motorischen Nerven des Ösophagus. Arch. f. d. ges. Physiol. Bd. 59. 
Kreidl ${ }^{1}$ ) hat nachgewiesen, dass die für die Muskulatur des Ösophagus bestimmten motorischen Fasern im oberen Wurzelbündel verlaufen.

Beer und Kreidla ${ }^{2}$ ) fanden im oberen Bündel Fasern, deren zentrale Reizung von Einfluss auf die Atmung ist.

Endlich hat Grossman ${ }^{3}$ ) im mittleren Bündel auch Fasern gefunden, welche die Hemmung des Herzens bewirken.

Durch die Uutersuchungen von Réthi wurden die Wurzelfasern für die Rachen- und Gaumenmuskulatur festgesetzt, wobei sich folgendes ergab: Die für den M. stylo-pharyngeus bestimmten Fasern verlaufen im oberen Bündel, die für die Konstriktoren des Rachens bestimmten Nerven und die Fasern des Levator vel. palatini gehören dem mittleren Bündel an.

Openchow $\mathrm{ski}^{4}$ ) beschreibt einen $\mathrm{N}$. dilatator cardiae, dessen Zentrum in dem Bezirk des Hirns sich befindet, wo sich das vordere untere Ende des Nucleus caudatus mit dem Nucleus lentiformis verbindet in geringer Entfernung von der vorderen Kommissur. Diese Fasern gesellen sich zu der Vagusbahn und verlaufen in beiden Vagis geteilt bis zum unteren Viertel des Ösophagus, an welcher Stelle sie sich sammeln, den Stamm der Vagi verlassen und an der Kardia endigen.

Die Aufgabe der vorliegenden Arbeit war es nun, jene Wurzelfasern zu bestimmen, welche für die Innervation des Magens und der Kardia in Betracht kommen.

Die Versuchsanordnung war die folgende: Das Tier (es wurde ausschliesslich an Kaninchen experimentiert) wurde narkotisiert, in Rückenlage die Membrana obturatoria freigelegt und gespalten. Hierauf wurde das Tier in Seitenlage gebracht und so viel von der Hinterhauptschuppe mit der Knochenzange abgetragen, bis das

1) Kreid1, Experimentelle Untersuchungen über das Wurzelgebiet des N. glossopharyngeus, Vagus und Accessorius. Sitzungsber. d. k. Akad. d. Wissensch. Bd. 106 Abt. III. 1897.

2) Th. Beer und Kreidl, Über den Ursprung der Vagusfasern, deren zentrale Reizung Verlangsamung resp. Stillstand der Atmung bewirkt. Pflüger's Arch. f. d. ges. Physiol. Bd. 62. 1895.

3) Grossmann, Über den Ursprung der Hemmungsnerven des Herzens. Pflüger's Arch. f. d. ges. Physiol. Bd. 59. 1894.

4) 0 penchowski, Über die nervösen Vorrichtungén des Magens. Zentralbl. f. Physiol. 1889 Nr. 1. 
mittels Stirnreflektor gut beleuchtete Versuchsfeld genau ubbersehen werden konnte. Lagen die drei Wurzelbündel genügend bloss und war das Gebiet frei von jeder Blutung, so wurde das Operationsfeld vorläufig mit Gaze verschlossen und an die Präparation des Magens geschritten. Während des Experimentes wurde der Magen häufig mit in warme Kochsalzlösung getauchten Kompressen befeuchtet.

Nun wurde die elektrische Reizung des Wurzelgebietes in der von Kreidl beschriebenen Weise vorgenommen, während ein Assistent sorgfältig die Bewegungen des Magens und der Kardia beobachtete. Die Reizung geschah in der Weise, dass die eine Elektrode mit dem Kopfhalter und dadurch mit dem Tier in leitende Verbindung gesetzt wurde, während die andere aus einem haarfeinen Platindraht bestehende Elektrode die einzelnen Wurzelfäden abtastete.

Gereizt wurde mit sehr schwachen Strömen eines $d u$ Boi s Reymond'schen Schlittenapparates, wobei die Fasern nur dann als bestimmt angenommen wurden, wenn die gleichen Ströme in den benachbarten Fasern keine Kontraktion der betreffenden Muskeln hervorriefen.

Versuch I.

Reizung des mittleren Bündels ruft eine langsame peristaltische Bewegung des präpylorischen Teiles des Magens hervor. Alle anderen Teile des Magens bleiben vollkommen bewegungslos.

Versuch II.

Reizung des oberen und des unteren Bündels bewirkt keine Bewegung des Magens.

Versuch III.

Der Accessorius mit dem unteren Bündel wird mittelst eines feinen Häkchens ausgerissen. Reizung des mittleren Bündels bewirkt Kontraktionen des präpylorischen Teiles des Magens.

\section{Versuch IV.}

Das obere und das untere Bündel werden ausgerissen, so dass bloss das mittlere Bündel stehen bleibt. Reizung desselben ergibt präpylorische Kontraktionen.

\section{Versuch V.}

Bei Reizung des oberen Bündels sieht man an der Einmündungsstelle des Ösophagus in den Magen eine blitzartig eintretende Kon- 
traktion des Ösophagus, welche beim Sistieren der elektrischen Reizung plötzlich aufhört.

Bei Reizung des mittleren und des unteren Bündels bleibt der Ösophagus vollkommen ruhig.

Nun wird der Magen längs der grossen Kurvatur aufgeschnitten und die lippenförmige Einmündung des Ösophagus in den Magen beobachtet, während eine Reizung des oberen Bündels erfolgt. Man sieht, dass sich die Lippen der Kardia plötzlich schliessen und nach Aufhören der Reizung wieder öffuen. Bei Reizung des mittleren und des unteren Bündels erfolgt keine Bewegung in der Einmündungsstelle des Ösophagus.

\section{Versuch VI.}

Es wird an dem Kaninchen die Ösophagotomie am Halse vorgenommen, ein Gummischlauch durch den Ösophagus bis zur Kardia vorgeschoben und der Schlauch mittelst Glastrichter mit Wasser gefüllt. Nun wird der Magen eröffnet und die Einmündungsstelle des Ösophagus in den Magen beobachtet. Man sieht, dass zeitweise der lippenförmige Verschluss des Ösophagus sich öffnet und Wasser aus dem Ösophagus in den Magen läuft, worauf wieder eine Kontraktion des Ösophagus eintritt und das Abfliessen des Wassers verhindert.

Nun wird das obere Bündel gereizt, worauf eine Kontraktiọn des Ösophagus eintritt, die so lange danert, als der Reiz ausgeübt wird. Beim Sistieren des Reizes öffnet sich die Kardia, und das Wasser stürzt aus dem Ösophagus in den Magen.

Aus den Versuchen V und VI konnte bei dem Charakter der Kontraktion der Cardia geschlossen werden, dass sich an dieser Stelle ein quergestreifter Schliesser und Öffner befinden müsse. Die Angaben der Literatur über die Muskelverbältnisse an der Einmündungsstelle des Ösophagus in den Magen lauten widersprechend.

Koelliker-Ebner ${ }^{1}$ ) fanden beim Menschen, dass in den zwei unteren Vierteilen des Ösophagus sich vorwiegend glatte Muskulatur vorfindet. Nach Ficinus finden sich bis zur Kardia einzelne quergestreifte Fasern, eine Angabe, welche Welcker und Schweigger-Seidel${ }^{2}$ ) und Klein ${ }^{3}$ ) nicht bestätigt fanden,

1) Koelliker, Handbuch der Gewebelehre des Menschen Bd. 3 S. 132. 1902.

2) Welcker und Schweigger-Seidel, Virchow's Arch. Bd. 21 S. 455.

3) Klein, Sitzungsber. d. k. Akademie d. Wissensch. Bd. 57. 1868. 
während in neuerer Zeit Coakley ${ }^{1}$ ) in der Gegend des Zwerchfellschlitzes, namentlich in der inneren Ringmuskelschicht reichliche, quergestreifte Fasern gefunden hat, die sich jedoch gegen die Kardia. bald verlieren.

Starling ${ }^{2}$ ) erwähnt, dass im oberen Teil des menschlichen Ösophagus quergestreifte und glatte Muskelfasern miteinander vorkommen, während der untere Teil zunächst dem Magen ausschliesslich aus glatten Muskeln besteht.

Auch ich fand bei der histologischen Untersuchung eines menschlichen Ösophagus, dass beim Übergang des Ösophagus in den Magen ausschliesslich glatte Muskulatur vorhanden ist.

Wesentlich andere Befunde erhielt ich bei der histologischen Untersuchung des Ösophagus der operierten Kaninchen. Ich konnte hierbei folgendes Verhalten der Muskulatur des Ösophagus bei seinem Fintritt in den Magen nachweisen.

Einige Zentimeter oberhalb der Kardia trifft man am Ösopbagus eine äussere und eine innere Schichte quergestreifter Längsmuskulatur, zwischen beiden Lagen befindet sich eine Schichte quergestreifter Ringmuskulatur. Auf Längeschnitten dureh den Ösophagus und die Kardia sieht man, dass beide Längsmuskelschichten gegen die Kardia zu allmählich immer schmäler werden und endlich ganz aufhören, während die Ringmuskulatur noch eine Strecke weit in die Kardia hinein sich erstreckt und keilförmig zwischen die daselbst bereits auftretenden glatten Muskelschichten des Magens vordringt (s. Taf. XIV Fig. 1).

Auf Querschnitten durch den Ösophagus in der Höhe des Durehtrittes durch das Zwerchfell sieht man eine äussere und eine innere Längsmuskelschichte und zwischen beiden eine Ringmuskelschichte, durchaus bestebend aus quergestreiften Muskelfasern (s. Taf. XIV Fig. 2).

Das Vorkommen dieser quergestreiften Muskelsehichten lässt es begreiflich erseheinen, dass in den Versuchen V und VI eine ruckartige, plötzliche Kontraktion der Kardia bei Reizung des oberen Bündels des Vagusstammes zustandekommt.

1) Coakley, Research. of the Loomis Labor. 1892 vol. 2.

2) E. H. Starling, Überblick über den gegenwärtigen Stand der Kenntnisse über die Bewegungen und die Innervation des Verdauungskanales. Ergebnisse der Physiologie 1902. 
Das Verhalten der aus der Medulla oblongata austretenden Nervenfasern ist schematisch durch die Fig. 1 veranschaulicht. Man sieht das obere Bündel des Vagus (-) gezeichnet, ein Teil des mittleren Bündels (-....-) zieht zur Kardia, ein Teil desselben Bündels (-.......) geht zur kleinen Kurvatur und zum Pylorus.

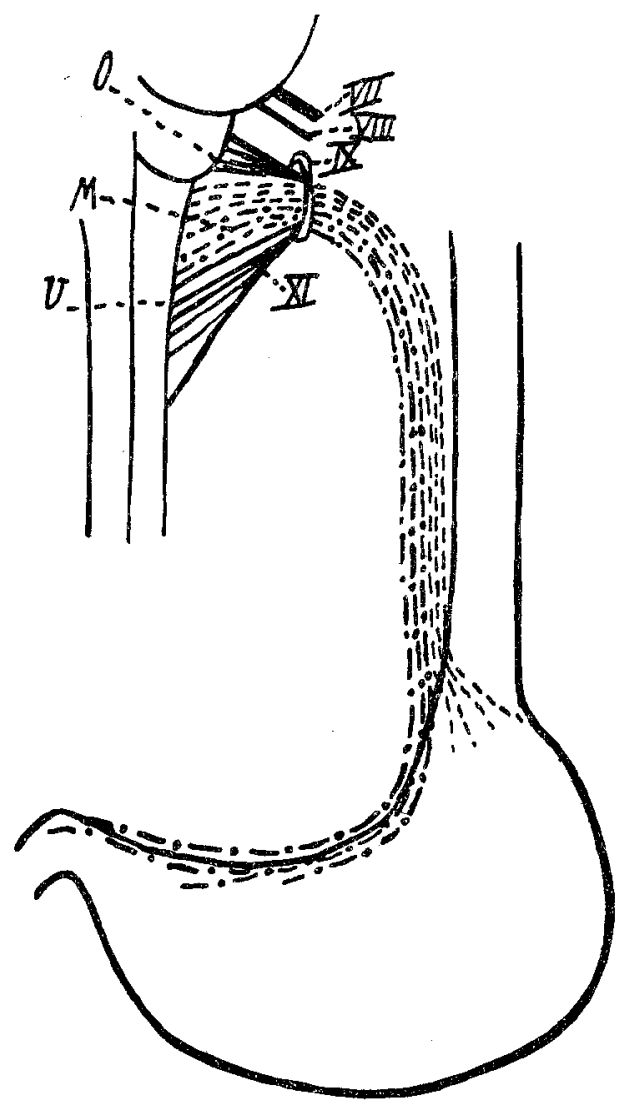

Fig. 1.

Zusammenfassend lassen sich aus den beschriebenen Versuchen folgende Schlüsse ziehen:

1. Das obere Bündel des aus der Medulla oblongata tretenden Vagus enthält Fasern für die Schliessung der Kardia.

2. Das mittlere Bündel des Vagusursprunges enthält die Fasern für die Bewegungen des präpylorischen Teiles des Magens.

3. Im untersten Teile des Ösophagus in der Gegend des Zwerchfellschlitzes befinden sich beim Kaninchen drei Schichten von quer- 
482 W. $Z$ weig: Die motor. Wurzelfasern für die Kardia und den Magen.

gestreifter Muskulatur, von welchen die äussere und die innerste Längsmuskeln und die mittlere eine Ringmuskelschichte darstellen.

Beim Menschen dagegen sind im untersten Abschnitt des Ösophagus quergestreifte Muskeln nicht vorhanden.

Zum Schlusse erfülle ich die angenehme Pflicht, Herrn Hofrat Professor Dr. Sigmund Exner und Herrn Professor Dr. Alois Kreidl für die stets bereite, werktätige Unterstützung bei der Ausführung meiner Versuche den ergebensten Dank auszudrücken. 